\title{
LapVac - a laparoscopic approach for negative pressure wound therapy application in open abdomen management: a porcine animal model
}

\author{
Adam Bobkiewicz, Maciej Borejsza-Wysocki, Lukasz Krokowicz, Tomasz Banasiewicz \\ Department of General, Endocrinological and Gastroenterological Oncology Surgery, Poznan University of Medical Sciences, Poznan, \\ Poland
}

Videosurgery Miniinv 2020; 15 (1): 112-116

DOI: https://doi.org/10.5114/wiitm.2019.86829

\begin{abstract}
Introduction: Negative pressure wound therapy (NPWT) in the open abdomen (OA) is recognized as a gold standard management method. Currently minimally invasive procedures are implemented in many clinical scenarios.

Aim: To demonstrate the feasibility of using negative pressure wound therapy in a laparoscopic approach for OA management in a porcine model termed as a laparoscopic vacuum (LapVac).

Material and methods: An adult female swine underwent a laparoscopic procedure. Briefly, a small incision was made and secured with a wound protector, pneumoperitoneum was created and two additional ports were placed. Then, a non-adhesive layer was precisely placed within the abdominal cavity.

Results: Finally, polyurethane foam and adhesive drape were applied. A volume of $200 \mathrm{ml}$ of saline solution was instilled and drained completely within 30 min. We did not observe any technical problems with NPWT application. Conclusions: This study confirmed the technical feasibility of NPWT application in the laparoscopic approach. LapVac seems to be a promising technique which may minimize the trauma and lead to better outcomes.
\end{abstract}

Key words: negative pressure wound therapy, laparoscopy, open abdomen.

\section{Introduction}

Negative pressure wound therapy (NPWT) in the open abdomen (OA) is recognized as a gold standard management. The strategy facilitates decompression and active drainage of intra-abdominal effluent, serves as a temporary abdominal closure and improves patient care and nursing [1]. Based on recent World Society of Emergency Surgery guidelines, $\mathrm{OA}$ is the management of choice in trauma patients and abdominal compartment syndrome [2]. The OA strategy also plays a crucial role in a selected group of patients complicated with severe pancreatitis and intra-abdominal sepsis [2].
However, OA management if applied excessively or inappropriately may be a source of severe side effects including intra-abdominal infection, loss of abdominal domain, development of enteroatmospheric fistulas and others [3].

Moreover, currently minimally invasive surgical procedures are implemented in many clinical scenarios. It results in lower postoperative patient morbidity and mortality when compared with a conventional approach. Thus, the purpose of this study was to demonstrate whether using NPWT in the laparoscopic approach in a porcine cadaver model is applicable for OA management. Patients suffering from intra-abdominal sepsis or severe pancreatitis 
require abdominal inspection as well as abdominal lavage. To minimize extensive laparotomy followed by prolonged laparostomy, we present an alternative to the standard approach. The presented technique, LapVac, may serve as a novel technique which allows a minimally invasive procedure for intra-abdominal inspection, decompression, lavage and facilitating rapid access for re-entry in patients without abdominal hypertension.

\section{Aim}

The authors hypothesized that the laparoscopic approach to NPWT application in OA management may: 1 ) reduce abdominal trauma, 2 ) preserve the abdominal wall domain, 3) decrease intra-abdominal adhesions and 4) optimize intra-abdominal lavage. To the best of our best knowledge, this is the first use of NPWT in a laparoscopic approach.

\section{Material and methods}

The experimental study was performed in a research laboratory (Aesculap Academy Nowy Tomysl, Poland). The animal was treated in accordance with the local guidelines for care and use of laboratory animals. The study was designed as a cadaver animal study. Thus, the swine was euthanized by a standard procedure directly before the experimental procedure.

A female 12-month-old swine weighing $55 \mathrm{~kg}$ underwent the experimental procedure.

\section{Results}

The pig was placed in a reverse Trendelenburg position. A 7-cm straight skin midline incision was made in the upper abdomen. Muscle and peritoneum were cut and the surgical approach was secured with a wound protector (Alexis O Wound Protector/ Retractor, Applied Medical, US) (Photo 1). Pneumoperitoneum of $12 \mathrm{~mm} \mathrm{Hg}$ using carbon dioxide was obtained. Under laparoscopic camera (Olympus, UK) guidance, two additional laparoscopic 5-mm ports were placed laterally to the second/third nipple interspaces on both sides (Photo 1). Inspection of the abdominal cavity was made.

A non-adhesive protective layer of NPWT (Abthera Open Abdomen Dressing, $\mathrm{KCl}$, US) was cut to the appropriate size (Photo 2) and inserted into the abdominal cavity through the wound protector (Photo 3). Using laparoscopic graspers, a non-adhe- sive protective layer was spread over the exposed organs within the abdominal cavity. Attention was paid to distribute it precisely in every quadrant of

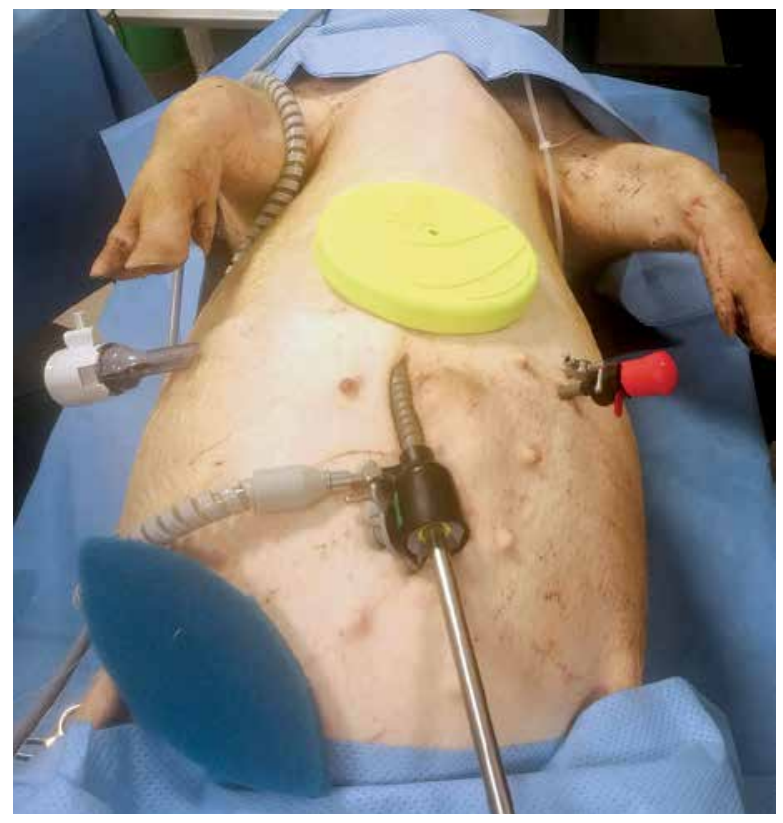

Photo 1. Swine positioning, surgical incision and locations of laparoscopic ports

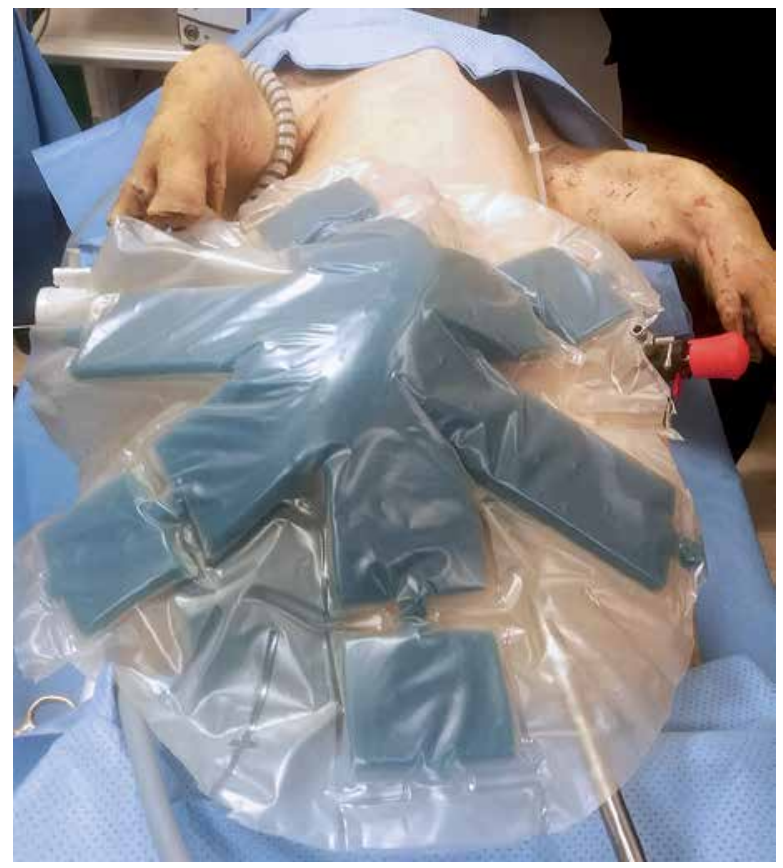

Photo 2. Preparation of non-adhesive protective layer. A protective layer trimmed to appropriate size with excised part of protruded polyurethane foam 


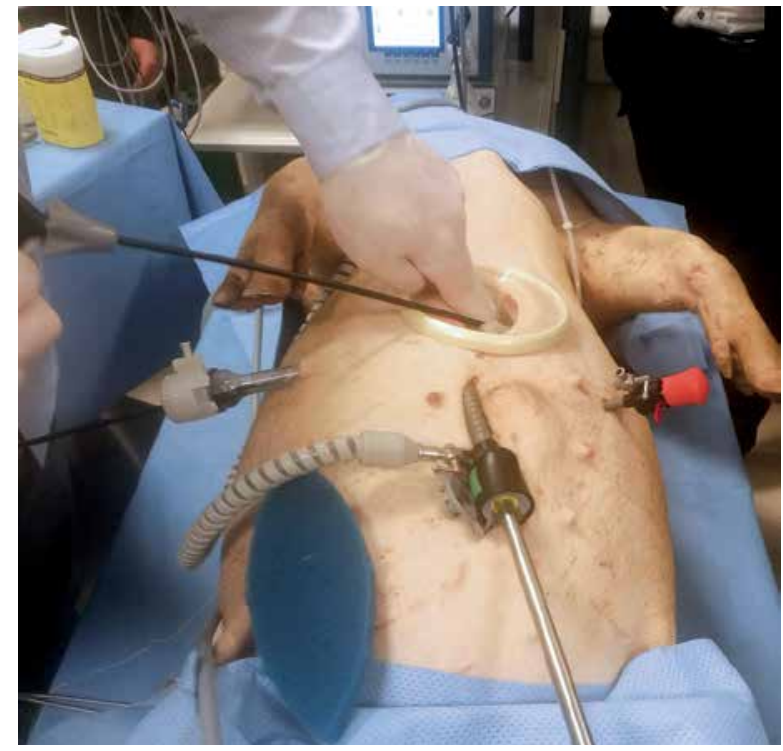

Photo 3. Insertion of a non-adhesive protective layer into the abdominal cavity

the abdominal cavity (Photo 4). The wound protector and laparoscopic ports were removed. A rectangular-shaped piece of polyurethane (PU) foam was applied over the non-adhesive protective layer to distribute negative pressure within the abdominal cavity. Additionally, another piece of PU foam was applied over the previous piece of PU foam stabilized with stomapaste (Stomahesive, Convatec, Poland) to keep the system sealed and to facilitate track-pad application. Then, an adhesive drape was applied

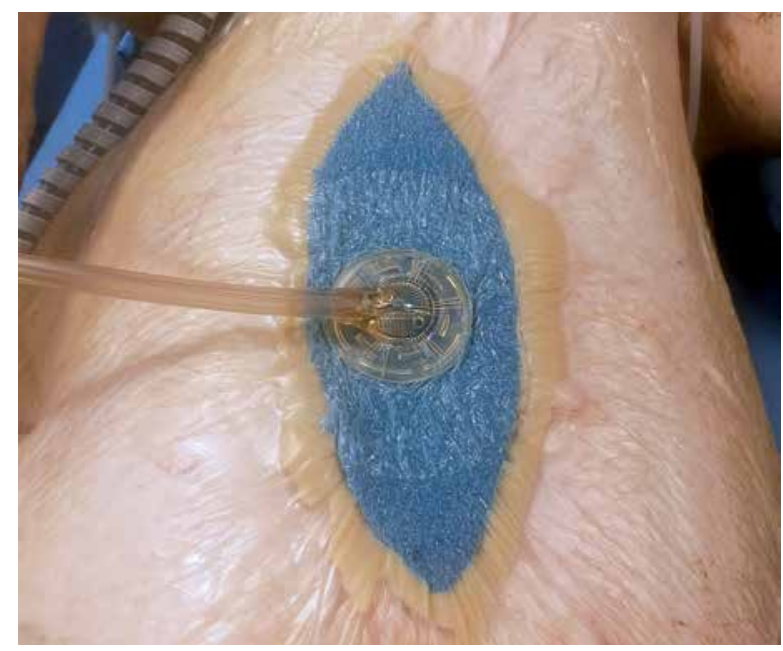

Photo 5. Polyurethane foams applied over the protective layer and stabilized with stomapaste. Application of adhesive drape and track-pad. NPWT sealed and set in continuous mode

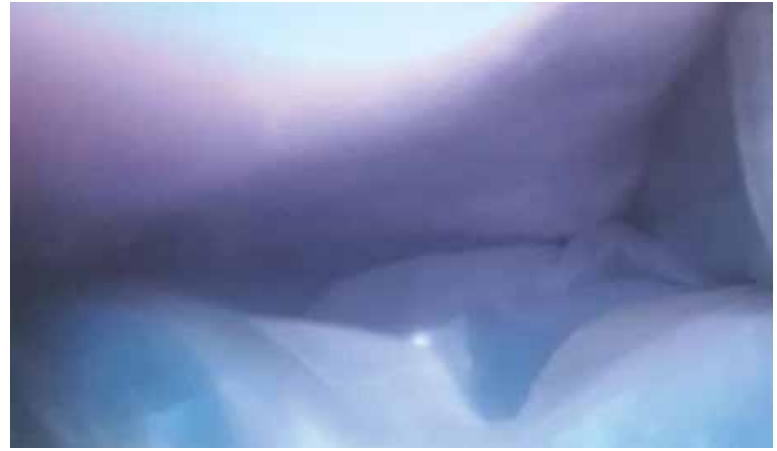

Photo 4. Intraoperative view. Non-adhesive protective layer placed precisely over the exposed abdominal organs

and $-80 \mathrm{~mm} \mathrm{Hg}$ was set in continuous mode (Photos 5 and 6). Skin incisions at port application sites were sutured with 3-0 non-absorbable sutures (Ethicon US, LLC).

Once NPWT was applied, $200 \mathrm{ml}$ of saline solution was instilled into the abdominal cavity. In $30 \mathrm{~min}$ the whole volume of saline was drained with the NPWT system. We did not observe any technical problems with NPWT application. Once the experimental study was accomplished, a wide abdominal incision was made. A non-adhesive sheet was applied properly without any migration or kinking.

\section{Discussion}

In the last decades, up to $15 \%$ of trauma laparotomies have been managed using OA [3]. Well-known problems with prolonged OA are development of lateralization of the abdominal wall, adhesions and frozen abdomen [1]. Moreover, fascial closure rates

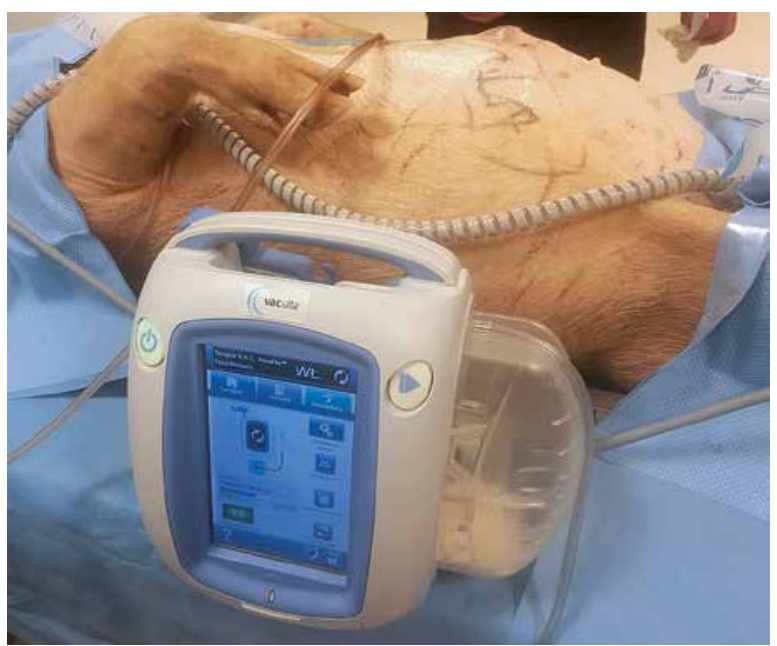

Photo 6. Seal check of NPWT 
are lower in non-trauma patients compared to trauma patients, with peritonitis being identified as an independent risk factor for failure of delayed fascial closure [4]. Finally, the rate of enteroatmospheric fistula formation is higher in patients with an OA due to peritonitis compared to non-septic patients [1].

This is the first porcine laparoscopic model for NPWT application that demonstrates the feasibility of using a minimally invasive procedure for open abdomen management.

Having considerable experience in use of NPWT in OA $[5,6]$, the authors hypothesized that LapVac may improve the strategy of OA. Anastomotic leakage, necrotizing pancreatitis, gastrointestinal perforation and bowel ischemia are the most common defined underlying pathologies of peritonitis required OA management [2]. Atema et al. in a systematic review and meta-analysis of OA reported a strategy of planned re-laparotomy and drainage of intra-abdominal sepsis as the most common indications for the necessity of leaving the abdominal cavity open [7]. In our opinion, reduction of laparostomy in size may help to prevent lateralization of the abdominal wall and delayed fascial closure. On the other hand, LapVac may be sufficient to achieve the goals of OA management. Every 48-hour intra-abdominal re-entry and maintenance of active drainage (optimally NPWT with instillation) fulfill the current concept of OA management. Following the current concept of early fascial closure in $\mathrm{OA}$, LapVac may serve as a method of choice for maintaining the integrity of the abdominal domain.

Laparoscopic camera guidance may accurately assess excavations of the peritoneal cavity and flush precisely the purulent or intestinal contents. In terms of abdominal inspection, placement of in tra-abdominal drainage or application of NPWT with instillation, LapVac may be a source of benefit in a selected group of patients.

From technical point of view, it is essential to cut the protective layer to an appropriate size, which may facilitate laparoscopic application. Positioning of a protective layer and grasping one of the corners of it before introduction into the abdominal cavity simplify the application.

Some disadvantages could be encountered using LapVac technique. First, appropriate selection of a patient for LapVac is crucial to obtain the benefit from such management. Second, lack of experience in open abdomen management in general may cause iatrogenic injuries. Thus, potentially LapVac may be limited to surgeons highly experienced in this method. The orientation of the protective layer within the abdominal cavity also requires some laparoscopic involvement and training.

In our experiment we demonstrated the feasibility of NPWT application using LapVac technique in OA. Introduction of NPWT for OA management in the late 1990s revolutionized this strategy. LapVac seems to be a promising technique which may minimize the OA trauma and favors better outcomes. However, potential benefits of this technique need to be supported by further experimental research and training in the porcine model to improve the physiologic and clinical relevance.

\section{Conclusions}

This study confirmed technical feasibility of NPWT application in the laparoscopic approach. This experimental study introduces the LapVac technique in a porcine model into the armamentarium of open abdomen research. Further in vivo studies in the porcine model are needed to investigate the concept of NPWT application in the laparoscopic approach. Moreover, we believe that potentially such a procedure may serve as an alternative to extensive standard open abdomen management in a selected group of patients.

\section{Conflict of interest}

Adam Bobkiewicz and Tomasz Banasiewicz have served as a consultant to $\mathrm{KCl}$, an Acelity Company.

Maciej Borejsza-Wysocki and Lukasz Krokowicz declare no conflict of interest.

\section{References}

1. Chiara O, Cimbanassi S, Biffl W, et al. International consensus conference on open abdomen in trauma. J Trauma Acute Care Surg 2016; 80: 173-83.

2. Coccolini F, Roberts D, Ansaloni L, et al. The open abdomen in trauma and non-trauma patients: WSES guidelines. World J Emerg Surg 2018; 13: 7.

3. Demetriades D, Salim A. Management of the open abdomen. Surg Clin North Am 2014; 94: 131-53.

4. van Boele HP, Wind J, Dijkgraaf MG, et al. Temporary closure of the open abdomen: a systematic review on delayed primary fascial closure in patients with an open abdomen. World J Surg 2009; 33: 199-207.

5. Bobkiewicz A, Walczak D, Smoliński S, et al. Management of enteroatmospheric fistula with negative pressure wound therapy in open abdomen treatment: a multicentre observational study. Int Wound J 2017; 14: 255-64. 
6. Bobkiewicz A, Banasiewicz T, Drews M. Postoperative pancreatic fistula successfully treated with "PEG-Like" endoscopic vacuum therapy. J Laparoendosc Adv Surg Tech A 2015; 25: 314-8.

7. Atema JJ, Gans SL, Boermeester MA. Systematic review and meta-analysis of the open abdomen and temporary abdominal closure techniques in non-trauma patients. World J Surg 2015; 39: 912-25.

Received: 29.04.2019, accepted: 6.06.2019. 